\title{
Entanglement and Closest Product States of Graph States with 9 to 11 Qubits
}

\author{
Cuifeng Wang, Lizhen Jiang, Lei Wang \\ College of Information and Electronic Engineering, Zhejiang, Gongshang University, Hangzhou, 310018, China \\ Email: wcf 0815@126.com
}

Received August 2013

\begin{abstract}
The numbers of local complimentary inequivalent graph states for 9, 10 and 11 qubit systems are 440,3132, 40457, respectively. We calculate the entanglement, the lower and upper bounds of the entanglement and obtain the closest product states for all these graph states. New patterns of closest product states are analyzed.
\end{abstract}

Keywords: Graph State; Entanglement; Closest Product State

\section{Introduction}

Graph states are certain pure multipartite quantum states associated to graphs [1-5] and have important applications in quantum error correction [6] and one-way quantum computer [7]. A variety of different entanglement measures have been proposed for multipartite quantum states, such as, the (Global) Robustness of Entanglement [8], the Relative Entropy of Entanglement [9,10], and the Geometric Measure [11]. Fortunately, these entanglement measures are all equal for stabilizer state [12]. It is known that the graph state is a subset of stabilizer state. Thus these entanglement measures are all equal for graph state. The geometric measure is the distance of the state to its closest product state in terms of the fidelity. The entanglement of graph states of 1 to 8 qubit systems has been calculated and the closest product states are found [13]. We will consider the entanglement of graph states of 9 to 11 qubits and their closest product states in this paper. Another closely related entanglement measure is the Schmidt measure [4,14].

\section{Preliminary}

The geometric measure of entanglement for pure state $|\psi\rangle$, is defined as

$$
E_{g}(|\psi\rangle)=\min _{|\phi\rangle \in \operatorname{Pro}}-\log _{2}|\langle\phi \mid \psi\rangle|^{2} .
$$

where Pro is the set of product states. A graph $G=(V ; \Gamma)$ is composed of a set $\mathrm{V}$ of $\mathrm{n}$ vertices and a set of edges specified by the adjacency matrix $\Gamma$, which is an $n \times n$ symmetric matrix with vanishing diagonal entries and $\Gamma_{a b}=1$ if vertices $a, b$ are connected and $\Gamma_{a b}=0$ otherwise. The graph state related to graph $G$ is defined as

$$
|G\rangle=\frac{1}{\sqrt{2^{n}}} \sum_{\mu=0}^{1}(-1)^{\frac{1}{2} \mu \Gamma \mu^{T}}|\mu\rangle_{z}
$$

where $0=(0,0, \ldots, 0)$ and $1=(1,1, \ldots, 1)$ are binary vectors of length $\mathrm{n}$. The entanglement of a graph state $|G\rangle$ can be written as

$$
E(|G\rangle)=E_{g}(|G\rangle)=\min _{\phi}-\log _{2}|\langle G \mid \phi\rangle|^{2}
$$

where $|\phi\rangle=\prod_{j}\left(\sqrt{p j}|0\rangle+\sqrt{1-p j} e^{i \varphi_{j}}|1\rangle\right)$ is the product pure state. All local complimentary (LC) equivalent graph states have the same amounts of entanglement, so only LC inequivalent graph states should be considered concerning with the entanglement. The number of LC inequivalent graph states increases with $\mathrm{n}$ rapidly. The number of LC inequivalent graph states up to 8 qubits is 146 , the entanglement is calculated with iterative method and the closest product states are found [13] and are denoted with $\left|\phi_{N o .1}\right\rangle$ to $\left|\phi_{\text {No.146 }}\right\rangle$.

The entanglement is upper bounded by the local operation and classical communication (LOCC) bound $E_{\text {LOCC }}=n-\log _{2} N$, where $N$ is the the maximal number of graph basis states that can be discriminated perfectly by LOCC $[13,15]$, and lower bounded by some bipartite entanglement deduced from the state, that is, the "matching" bound $E_{b i}[16]$. Thus

$$
E_{b i} \leq E(|G\rangle) \leq E_{L O G G}
$$

For a large number of graph states considered later, we have $E_{b i}=E_{L O G G}$, the entanglement can be easily be determined. While for graph states with unequal lower 
and upper bounds, we will use iterative method [13] to calculate the entanglement and obtain the closest product states.

\section{Classifications of the Graph States of Nine, Ten and Eleven Qubits}

All the graphs of 9, 10 and 11 vertices that are LC inequivalent for their graph states can be found in Ref. [17]. The total number of inequivalent classes of 9-qubit graph states is 440 . We denote them from No.147 to No.586. The entanglement of the graph states is classified and listed in Table 1. The entanglement of graph states with equal lower and upper bounds can be calculated with the methods in Ref [16], and we use computer program to determine the bounds. The entanglement of graph states with unequal bounds can be calculated with the iterative algorithm [13].

These two graph states whose entanglement values is 5.5124 and 5.8381 are new in the sense that they have different values of entanglement after the decimal point with respect to the former (1 to 8 qubit) graph states.

The total number of inequivalent classes of 10-qubit graph states is 3132 , and we denote the graphs from No.587 to No.3718. The entanglement is classified and listed in Table 2.

The total number of inequivalent classes of 11-qubit graph states is 40457 , and we denote them from No.3719 to No.44175. The entanglement is classified and listed in Table 3.

\section{Structures of the Closest Product States of Nine, Ten and Eleven Qubits}

\subsection{The Nine Qubit System}

Denote $\left|\phi_{j}\right\rangle=\sqrt{p}|0\rangle+\sqrt{1-p} e^{i \varphi_{j}}|1\rangle,(j=1, \ldots, 4)$, with $\sqrt{p}=\sqrt{\frac{1}{2}\left(1-\frac{1}{\sqrt{3}}\right)}=0.4597, \varphi_{1}=\frac{\pi}{4}, \varphi_{2}=-\frac{\pi}{4}, \varphi_{3}=\frac{3 \rho}{4}$, $\varphi_{4}=-\frac{3 \rho}{4} ; \quad| \pm\rangle=\frac{1}{\sqrt{2}}(|0\rangle \pm|1\rangle) ;|O\rangle=\frac{1}{\sqrt{2}}(|0\rangle-i|1\rangle)$, $\left|O^{\prime}\right\rangle=\frac{1}{\sqrt{2}}(|0\rangle+i|1\rangle)$. The closest product state of ring 5 graph state is $\left|\phi_{\text {ring } 5}\right\rangle=\left|\phi_{\text {No.8 }}\right\rangle=\left|\phi_{1}\right\rangle^{\otimes 5}$, the entanglement is $E_{\text {No. } 8}=1+\log _{2} 3+\log _{2}(3-\sqrt{3}) \approx 2.9275 \quad$ [13]. The graph set with non-integer entanglement $(\mathrm{k}+0.9275)$ graph states is closely related with ring 5 graph. The closest product state of graph state whose entanglement equals 3.9275 can be

$$
|\phi\rangle=|+\rangle^{\otimes 3}\left|\phi_{4}\right\rangle^{\otimes 2}\left|\phi_{1}\right\rangle^{\otimes 3}|0\rangle
$$

The closest product state of graph state whose entan-
Table 1. Classification of the graph state of 9 qubits.

\begin{tabular}{cccc}
\hline$E$ & $E_{b i}$ & $E_{\text {locc }}$ & NUM \\
\hline 1 & 1 & 1 & 1 \\
2 & 2 & 2 & 10 \\
3 & 3 & 3 & 61 \\
3.9275 & 3 & 4 & 3 \\
4 & 4 & 4 & 207 \\
4.5850 & 4 & 5 & 2 \\
4.9275 & 4 & 5 & 42 \\
5 & 4 & 5 & 112 \\
5.5124 & 4 & 6 & 1 \\
5.8381 & 4 & 6 & 1 \\
\hline
\end{tabular}

Table 2. Classification of the graph state of 10 qubits.

\begin{tabular}{cccc}
\hline$E$ & $E_{b i}$ & $E_{\text {locc }}$ & $N U M$ \\
\hline 1 & 1 & 1 & 1 \\
2 & 2 & 2 & 11 \\
3 & 3 & 3 & 103 \\
3.9275 & 3 & 4 & 3 \\
4 & 4 & 4 & 631 \\
4 & 4 & 5 & 5 \\
4.5850 & 4 & 5 & 2 \\
4.9275 & 4 & 5 & 76 \\
5 & 5 & 5 & 1536 \\
5 & 4 & 5 & 100 \\
5.5850 & 4 & 6 & 1 \\
5.9275 & 4 & 6 & 13 \\
6 & 4 & 6 & 13 \\
5 & 5 & 6 & 40 \\
5.5850 & 5 & 6 & 28 \\
5.8549 & 5 & 6 & 12 \\
5.9275 & 5 & 6 & 217 \\
6 & 5 & 6 & 339 \\
6.1669 & 5 & 7 & 1 \\
\hline & & &
\end{tabular}

glement equals 4.9275 can be

$$
|\phi\rangle=|+\rangle^{\otimes 3}\left|\phi_{4}\right\rangle^{\otimes 2}\left|\phi_{1}\right\rangle^{\otimes 3}|0\rangle
$$

The graph set with non-integer entanglement $(\mathrm{k}+$ $0.5850)$ graph states is closely related with No.19 graph. The closest product state of graph state whose entanglement equals 4.5850 can be

$$
|\phi\rangle=|+\rangle^{\otimes 2}\left|\phi_{3}\right\rangle^{\otimes 5}|0\rangle\left|\phi_{1}\right\rangle
$$

No.510 has a new structure in 9-qubit graph states. Its entanglement is

$$
E_{\text {No. } 510}=2+2 \log _{2}(3)+\log _{2}(3-\sqrt{3}) \approx 5.5124
$$

its closest product state can be 
Table 3. Classification of the graph state of 11 qubits.

\begin{tabular}{|c|c|c|c|}
\hline$E$ & $E_{b i}$ & $E_{\text {locc }}$ & NUM \\
\hline 1 & 1 & 1 & 1 \\
\hline 2 & 2 & 2 & 13 \\
\hline 3 & 3 & 3 & 163 \\
\hline 3.9275 & 3 & 4 & 3 \\
\hline 4 & 4 & 4 & 1561 \\
\hline 4 & 4 & 5 & 14 \\
\hline 4.5850 & 4 & 5 & 2 \\
\hline 4.9275 & 4 & 5 & 121 \\
\hline 5 & 5 & 5 & 9951 \\
\hline 5 & 5 & 6 & 286 \\
\hline 5 & 4 & 5 & 125 \\
\hline 5.5850 & 4 & 6 & 1 \\
\hline 5.5850 & 5 & 6 & 48 \\
\hline 5.8549 & 5 & 6 & 12 \\
\hline 5.9275 & 5 & 6 & 1936 \\
\hline 5.9275 & 5 & 7 & 1 \\
\hline 5.9275 & 4 & 6 & 2 \\
\hline 6 & 5 & 6 & 22573 \\
\hline 6 & 5 & 7 & 351 \\
\hline 6.5124 & 5 & 7 & 4 \\
\hline 6.5850 & 5 & 7 & 67 \\
\hline 6.7824 & 5 & 7 & 1 \\
\hline 6.8381 & 5 & 7 & 1 \\
\hline 6.8549 & 5 & 7 & 35 \\
\hline 6.9275 & 5 & 7 & 1145 \\
\hline 7 & 5 & 7 & 2040 \\
\hline
\end{tabular}

$$
\left|\phi_{\text {No.510 }}\right\rangle=\left|\phi_{2}\right\rangle^{\otimes 9} \text {. }
$$

The entanglement of No.582 is 5.8381, and its closest product state has a totally new structure. The known closest product state include components $\left|\phi_{j}\right\rangle(j=1, \cdots, 4),|0\rangle,|1\rangle,|O\rangle,\left|O^{\prime}\right\rangle$, however, there are 5 new components in the closest product state of No.582.

Denote $\left|\phi_{j}\right\rangle=\sqrt{p_{j}}|0\rangle+\sqrt{1-p_{j}} e^{i \varphi_{j}}|1\rangle,(j=5, \cdots, 9)$.

The new components are listed as follows,

$$
\begin{aligned}
& \left|\phi_{5}\right\rangle: \sqrt{p} \approx 0.9357, \varphi= \pm 7.3^{\circ}, \\
& \left|\phi_{6}\right\rangle: \sqrt{p} \approx 0.9255, \varphi= \pm 21.4^{\circ}, \\
& \left|\phi_{7}\right\rangle: \sqrt{p} \approx 0.4365, \varphi= \pm 43.0^{\circ}, \\
& \left|\phi_{8}\right\rangle: \sqrt{p} \approx 0.4208, \varphi= \pm 44.7^{\circ}, \\
& \left|\phi_{9}\right\rangle: \sqrt{p} \approx 0.8807, \varphi= \pm 43.8^{\circ} .
\end{aligned}
$$

In fact, we can find 15 kinds of new component qubits in the closest product state of graph state No.582. However, we can transform these 15 new component qubits into the above five kinds of qubits by using unitary transformation $U_{1}=\frac{1}{\sqrt{2}}\left(\begin{array}{cc}1 & 1 \\ -1 & 1\end{array}\right)$ or $U_{2}=\frac{1}{\sqrt{2}}\left(\begin{array}{ll}1 & i \\ i & 1\end{array}\right)$. Using iterative algorithm, we find the closest product states of graph state No.582, and denote them as

$$
\begin{aligned}
& \left|\Phi_{1}\right\rangle=E D D B C A A B C, \\
& \left|\Phi_{2}\right\rangle=D E A A C B D C B, \\
& \left|\Phi_{3}\right\rangle=D A E C B D B A C, \\
& \left|\Phi_{4}\right\rangle=B A C E A C D B D, \\
& \left|\Phi_{5}\right\rangle=C C B A E A B D D, \\
& \left|\Phi_{6}\right\rangle=A B D C A E C D B, \\
& \left|\Phi_{7}\right\rangle=A D B D B C E C A, \\
& \left|\Phi_{8}\right\rangle=B C A B D D C E A, \\
& \left|\Phi_{9}\right\rangle=C B C D D B A A E .
\end{aligned}
$$

where A, B, C, D, E represent $\left|\phi_{5}\right\rangle, \cdots,\left|\phi_{9}\right\rangle$, respectively. If we ignore the order of qubits, the closest product state of graph state No.582 can be written as

$$
\left|\phi_{N o .582}\right\rangle=E A^{\otimes 2} B^{\otimes 2} C^{\otimes 2} D^{\otimes 2}
$$

\subsection{The Ten Qubit System}

In Table 2, the graph set with non-integer entanglement $(\mathrm{k}+0.9275)$ graph states is characterized by ring 5 graph. The closest product state can be

$$
|\phi\rangle=|+\rangle^{\otimes 4}\left|\phi_{2}\right\rangle^{\otimes 5}|0\rangle
$$

for ten qubit graph state with entanglement 3.9275, it can be

$$
|\phi\rangle=|+\rangle^{\otimes 3}\left|\phi_{3}\right\rangle^{\otimes 3}\left|\phi_{1}\right\rangle^{\otimes 2}|0\rangle^{\otimes 2}
$$

for graph state with entanglement 4.9275, it can be

$$
|\phi\rangle=|+\rangle^{\otimes 2}\left|\phi_{3}\right\rangle^{\otimes 3}|0\rangle^{\otimes 2}\left|\phi_{2}\right\rangle|0\rangle\left|\phi_{3}\right\rangle
$$

when with entanglement 5.9275. The graph set with noninteger entanglement $(k+0.8549)$ graph states is characterized by graph state No.133. The entanglement of graph state No. 133 is

$E_{\text {No.133 }}=1+2 \log _{2} 3+2 \log _{2}(3-\sqrt{3}) \approx 4.8549$, its closest product state can be $\left|\phi_{\text {No133 }}\right\rangle=\left|\phi_{4}\right\rangle\left|\phi_{1}\right\rangle^{\otimes 2}\left|\phi_{4}\right\rangle^{\otimes 2}\left|\phi_{1}\right\rangle^{\otimes 2}\left|\phi_{4}\right\rangle$ [13]. The closest product state of graph state whose entanglement equals 5.8549 can be

$$
|\phi\rangle=|+\rangle\left|\phi_{3}\right\rangle^{\otimes 2}\left|\phi_{2}\right\rangle\left|\phi_{1}\right\rangle\left|\phi_{3}\right\rangle^{\otimes 2}\left|\phi_{2}\right\rangle\left|\phi_{3}\right\rangle|0\rangle
$$

The only ten qubit graph state with new pattern of closest product state is graph state No.3599 whose en- 
tanglement is

$$
E_{\text {No. } 3599}=3+2 \log _{2} 3 \approx 6.1699
$$

the closest product state can be

$$
\left|\phi_{\text {No.3599 }}\right\rangle=\left|\phi_{3}\right\rangle\left|\phi_{4}\right\rangle^{\otimes 2}\left|\phi_{3}\right\rangle^{\otimes 5}\left|\phi_{1}\right\rangle^{\otimes 2}
$$

\subsection{The Eleven Qubit System}

For eleven qubit graph states show in Table 3, a detail comparison of computed closest product states of No.3724, No.3765 shows that all these closest states have a substructure of the closest product state of ring 5 graph. Ring 5 graph is essential to all these graph states with entanglement $\mathrm{k}+0.9275$ (integer $\mathrm{k}$ ). The closest product states can be

$$
\begin{array}{r}
\left|\phi_{\text {No. } 3724}\right\rangle=|+\rangle^{\otimes 5}\left|\phi_{2}\right\rangle\left|\phi_{3}\right\rangle^{\otimes 4}|0\rangle \\
\left|\phi_{N o .3765}\right\rangle=|-\rangle^{\otimes 4}\left|\phi_{2}\right\rangle\left|\phi_{4}\right\rangle^{\otimes 2}|1\rangle\left|\phi_{2}\right\rangle^{\otimes 2}|0\rangle
\end{array}
$$

The graph set (No.3764, No.3936) with non-integer entanglement $(\mathrm{k}+0.5850)$ graph states is characterized by graph No.19. The entanglement of graph state No.19 is $E_{\text {No. } 19}=2+\log _{2} 3 \approx 3.5850$ Typically, the closest product state is $\left|\phi_{N o .19}\right\rangle=\left|\phi_{3}\right\rangle^{\otimes 3}\left|\phi_{4}\right\rangle^{\otimes 3} \quad$ [13]. The closest state for No.3764 and No.3936 graph state can be

$$
\begin{array}{r}
\left|\phi_{\text {No. } 3764}\right\rangle=|+\rangle^{\otimes 4}\left|\phi_{2}\right\rangle\left|\phi_{3}\right\rangle\left|\phi_{2}\right\rangle\left|\phi_{3}\right\rangle^{\otimes 2}|0\rangle\left|\phi_{1}\right\rangle \\
\left|\phi_{\text {No. } 3936}\right\rangle=|+\rangle^{\otimes 3}|0\rangle\left|\phi_{3}\right\rangle\left|\phi_{2}\right\rangle\left|\phi_{4}\right\rangle|0\rangle\left|\phi_{4}\right\rangle^{\otimes 2}\left|\phi_{2}\right\rangle
\end{array}
$$

respectively. The graph set (No.4113, No.30597) with non-integer entanglement $(\mathrm{k}+0.8549)$ graph states is characterized by graph state No.133. The closest state for No.4113 and No.30597 graph state can be

$$
\begin{gathered}
\left|\phi_{\text {No.4113 }}\right\rangle|+\rangle^{\otimes 2}\left|\phi_{1}\right\rangle\left|\phi_{3}\right\rangle\left|\phi_{1}\right\rangle\left|\phi_{4}\right\rangle\left|\phi_{1}\right\rangle\left|\phi_{4}\right\rangle^{\otimes 3}|0\rangle, \\
\left|\phi_{\text {No. } 30597}\right\rangle=\left|\phi_{1}\right\rangle^{\otimes 2}|+\rangle\left|\phi_{1}\right\rangle|0\rangle\left|\phi_{1}\right\rangle^{\otimes 2}|0\rangle\left|\phi_{1}\right\rangle\left|\phi_{4}\right\rangle^{\otimes 2} .
\end{gathered}
$$

The entanglement of No.30505 is a new type in 11 -qubit graph states. Its entanglement is

$$
E_{\text {No. } 30505}=2+3 \log _{2}(3-\sqrt{3}) \approx 6.7824
$$

its closest product state can be

$$
\left|\phi_{\text {No.30505 }}\right\rangle=\left|\phi_{1}\right\rangle\left|\phi_{4}\right\rangle\left|\phi_{1}\right\rangle\left|\phi_{4}\right\rangle\left|\phi_{1}\right\rangle\left|\phi_{4}\right\rangle^{\otimes 4}\left|\phi_{1}\right\rangle^{\otimes 2}
$$

The entanglement of No.23813 is 6.8381, the closest product state of No.23813 can be

$$
\left|\phi_{\text {No.23813 }}\right\rangle=|+\rangle B E D B|1\rangle D C A A C
$$

It contains the closest product of graph state No.582 $\left|\phi_{\text {No.582 }}\right\rangle$ as its substructure.

Note that, we have just found one of the many closest product states for each LC inequivalent graph state. Actually, there are many local equivalent closest product states for the same graph state.

\section{Conclusions}

We calculate the entanglement of all local complimentary inequivalent graph states with nine, ten and eleven qubits. The total number of the graph states we treated is 44029, and we list the numbers of them in Tables 1-3 according to their entanglement. Further calculation for twelve qubits is quite difficult since there are more than 1.27 million of local complimentary inequivalent graph states. Four new types of non-integer entanglement values appear in 9, 10 and 11 qubit graph states. The detail results and some special characters of the closest product states are as follows: (1) The graph sets with non-integer entanglement $(\mathrm{k}+0.9275, \mathrm{k}+0.5850$ and $\mathrm{k}+0.8549)$ graph states are specified by ring 5 graph, No.19 graph and No.133 graph, respectively. Their closest product states contain five, six and eight $\left|\phi_{j}\right\rangle(j=1, \cdots, 4) ;(2)$ The closest product states of graph states with non-integer entanglement (5.5124 for nine qubits, 6.1669 for ten qubits, 6.7824 for eleven qubits) contains 9, 10 and $11\left|\phi_{j}\right\rangle(j=1, \cdots, 4)$, respectively; (3) The graph states with non-integer entanglement $(\mathrm{k}+0.8381)$ have a new structure in their closest product states. We find 5 new components $\left|\phi_{j}\right\rangle(j=5, \cdots, 9)$ in the closest product states. (4). The closest product state for graph state with integer entanglement does not contain $\left|\phi_{j}\right\rangle(j=5, \cdots, 9)$.

\section{Acknowledgements}

Funding by the National Natural Science Foundation of China (Grant No. 60972071), Natural Science Foundation of Zhejiang Province (Grant No. Y6100421), Zhejiang Province Science and Technology Project (Grant No. 2009C31060) are gratefully acknowledged.

\section{REFERENCES}

[1] R. Raussendorf, D. E. Browne and H. J. Briegel, "Measurement-Based Quantum Computation on Cluster States," Physical Review A, Vol. 68, No. 2, 2003, Article ID: 022312. http://dx.doi.org/10.1103/PhysRevA.68.022312

[2] D.-M. Schlingemann, Quant. Inf. Comp., Vol. 2, 2002, p. 307.

[3] D.-M. Schlingemann, Quant. Inf. Comp., Vol. 4, 2002, p. 287.

[4] M. Hein, J. Eisert and H. J. Briegel, "Multiparty Entanglement in Graph States," Physical Review A, Vol. 69, No. 6, 2004, Article ID: 062311. http://dx.doi.org/10.1103/PhysRevA.69.062311

[5] M. Hein, W. Dur, J. Eisert, R. Raussendorf, M. Van den Nest and H. J. Briegel, In G. Casati, D. L. Shepelyansky, P. Zoller and G. Benenti, Eds., Quantum Computers, Algorithms and Chaos, IOS Press, Amsterdam, 2006.

[6] M. Grassl, A. Klappenecker and M. Rotteler, "Graphs, 
Quadratic Forms, and Quantum Codes," Proceedings of 2002 IEEE International Symposium on Information Theory, Lausanne, Switzerland, p. 45. http://dx.doi.org/10.1109/ISIT.2002.1023317

[7] R. Raussendorf and H. J. Briegel, "A One-Way Quantum Computer," Physical Review Letters, Vol. 86, No. 22, 2001, pp. 5188-5191. http://dx.doi.org/10.1103/PhysRevLett.86.5188

[8] G. Vidal and R. Tarrach, "Robustness of Entanglement," Physical Review A, Vol. 59, No. 1, 1999, pp. 141-150. http://dx.doi.org/10.1103/PhysRevA.59.141

[9] V. Vedral, M. B. Plenio, M. A. Rippin and P. L. Knight, "Quantifying Entanglement," Physical Review Letters, Vol. 78, No. 12, 1997, pp. 2275-2279. http://dx.doi.org/10.1103/PhysRevLett.78.2275

[10] V. Vedral and M. B. Plenio, "Entanglement Measures and Purification Procedures," Physical Review A, Vol. 57, No. 3, 1998, pp. 1619-1633. http://dx.doi.org/10.1103/PhysRevA.57.1619

[11] T.-C. Wei and P. M. Goldbart, "Geometric Measure of Entanglement and Applications to Bipartite and Multipartite Quantum States," Physical Review A, Vol. 68, No. 4, 2003, Article ID: 042307. http://dx.doi.org/10.1103/PhysRevA.68.042307

[12] M. Hayashi, D. Markham, M. Murao, M. Owari and S.
Virmani, "Entanglement of Multiparty-Stabilizer, Symmetric, and Antisymmetric States," Physical Review A, Vol. 77, No. 1, 2008, Article ID: 012104. http://dx.doi.org/10.1103/PhysRevA.77.012104

[13] X. Y. Chen, "Entanglement of Graph States up to Eight Qubits," Journal of Physics B, Vol. 43, No. 8, 2010, Article ID: 085507. http://dx.doi.org/10.1088/0953-4075/43/8/085507

[14] A. Cabello, A. J. Lopez-Tarrida, P. Moreno and J. R. Portillo, "Entanglement in Eight-Qubit Graph States," Physics Letters A, Vol. 373, No. 26, 2009, pp. 2219-2225. http://dx.doi.org/10.1016/j.physleta.2009.04.055

[15] M. Hayashi, D. Markham, M. Murao, M. Owari and S. Virmani, "Bounds on Multipartite Entangled Orthogonal State Discrimination Using Local Operations and Classical Communication," Physical Review Letters, Vol. 96, No. 4, 2006, Article ID: 040501. http://dx.doi.org/10.1103/PhysRevLett.96.040501

[16] D. Markham, A. Miyake and S. Virmani, "Entanglement and Local Information Access for Graph States," New Journal of Physics, Vol. 9, 2007, pp. 194. http://dx.doi.org/10.1088/1367-2630/9/6/194

[17] L. E. Danielsen, "Database of Self-Dual Quantum Codes." http://www.ii.uib.no/larsed/vncorbits/ 